\title{
Laparoscopic versus open surgery for gastric cancer: the experience of one European centre
}

\author{
Mindaugas Kiudelis, Aistė Rikterė, Kristina Zviniene, Antanas Mickevicius, Almantas Maleckas, \\ Audrius Ivanauskas, Zilvinas Endzinas \\ Department of Surgery, Gastroenterology and Radiology, Lithuanian University of Health Sciences, Kaunas, Lithuania
}

Gastroenterology Rev 2021; 16 (2): 174-180

DOI: https://doi.org/10.5114/pg.2021.106670

\begin{abstract}
Address for correspondence: Prof. Mindaugas Kiudelis, Department of Surgery, Gastroenterology and Radiology, Lithuanian University of Health Sciences, Kaunas, Lithuania, phone: +37 068757903, e-mail: minkiud@gmail.com
\end{abstract}

Gastric cancer is the third leading cause of cancer death and the fifth most common cancer worldwide: more than a million new cases of gastric cancer are diagnosed every year, with more than 700,000 deaths each year [1, 2]. It causes 1 out of 12 oncological disease-related deaths worldwide. The highest number of new cases is observed in Eastern Asian and Eastern European countries. While incidence rates of gastric cancer in North America, Africa, and South and West Asia are declining, rates in North-East Asia, the Eastern part of South America and Eastern Europe remain high [3]. In Europe, mortality from gastric cancer ranks the fourth among oncological diseases. According to the Lithuanian Cancer Registry, around 1000 people get gastric cancer in this country every year, and over 700 die from relative causes [4]. The 5 -year survival rate for patients with this disease is only $25 \%$ [4].

Surgery has remained the mainstay of the curative approach for gastric cancer for a long time. Following ESMO Clinical Practice Guidelines [5], endoscopic tumour removal is recommended for stage IA gastric cancer with a T1N0 tumour of size $\leq 2 \mathrm{~cm}$. According to the Japanese Gastric Cancer Association treatment guidelines [6], in locally advanced gastric cancer CT1$4 \mathrm{~N}+\mathrm{MO}$, D2 lymphadenectomy with radical surgery is recommended. Currently, D2 lymphadenectomy is considered a standard procedure in Western Europe and in Lithuania.

With growing surgical experience, minimally invasive surgical methods are gaining ground. According to various references [7-9], laparoscopic total or subtotal gastrectomy in locally advanced gastric cancer has advantages over open surgery due to less surgical trauma, leading to faster recovery and potentially earlier adjuvant chemotherapy. According to this data, the incidence and outcome of postoperative complications as well as the postoperative mortality rate do not differ significantly between open and laparoscopic surgical methods. On the other hand, most of these data comes from East Asian countries (China, Japan, South Korea), but there are not so many publications comparing laparoscopic versus open advanced gastric cancer surgery results among Western or Eastern European populations.

The aim of this study was to compare the shortand long-term treatment results between radical laparoscopic and open surgeries for advanced gastric cancer in a single high-volume gastric cancer surgery centre.

This was a retrospective non-randomized, single-centre, cohort study. Data collection was performed at the Clinic of Surgery of University Hospital using a specially developed and maintained database from 1.01.2013 to 31.12.2017. All patients gave their written informed consent, and the local Ethics committee approved the study (No.BE-2-66). Gastric carcinoma in all cases was preoperatively confirmed by a pathological examination of endoscopic biopsy samples. Chest, abdomen, and pelvic computed tomography (CT) examination was performed on all included patients before surgery, in order to determine the clinical diagnosis and to rule out possible distant disease spread. The treatment plan for each patient was approved by a multidisciplinary team. Four experienced upper gastrointestinal tract surgeons performed all open and laparoscopic operations. Standard total (adenocarcinoma involving the proximal third of the stomach) or subtotal (adenocarcinoma of the distal and middle thirds of the stomach) gastrectomy with D2 lymphadenectomy and a Rouxen-Y reconstruction was performed according to the Japanese Gastric Cancer Treatment Guidelines [6, 10]. The choice of partial or total gastrectomy was made depending on the possibility to achieve an adequately 
distant proximal resection margin $(\geq 6 \mathrm{~cm})$. Pathological stages were classified according to the Japanese Classification of Gastric Carcinoma [10]. The type of surgery (open or laparoscopic) was chosen independently by the operating surgeon.

The same postoperative treatment protocols were applied to all participating patients. The decision regarding an appropriate timing for starting a soft diet as well as patients' discharge time was finally decided by the operating surgeon in accordance with the patients' conditions.

During this period 175 patients underwent radical surgery for gastric cancer. One hundred and thirty-six consecutive patients were included in this study, according to the following inclusion criteria: (1) histologically proven gastric adenocarcinoma; (2) diagnosis based on the UICC TNM staging classification; (3) curative D2 performed; (4) available complete medical record; and (5) postoperative follow-up.

The study population was divided into 2 groups according to the type of surgery: The first group, comprising 96 patients, underwent open radical total or subtotal gastrectomy (ORG) + D2 lymphadenectomy The second group, comprising 40 patients, underwent laparoscopic radical total or subtotal gastrectomy (LRG) + D2 lymphadenectomy.

ORG was performed according to our standard protocol through an upper-middle incision. All patients underwent antecolic Roux-en-Y reconstruction. After removal of the stomach, end-to-side oesophagojejunostomy was performed using a circular stapler $(\mathrm{CDH}$, $25 \mathrm{~mm}$; Ethicon Endo-Surgery or CEEA, $25 \mathrm{~mm}$; Covidien) for open gastrectomy. End-to-side jejunojejunal anastomosis was performed approximately $40 \mathrm{~cm}$ below the oesophagojejunal junction using continuous single-layer sutures. For open subtotal gastrectomy, gastrojejunostomy and end-to-side jejunojejunal anastomosis were performed with continuous single-layer sutures.

LRG was performed using the 5 trocars technique. All reconstructions were done intracorporeally. All patients underwent antecolic Roux-en-Y reconstruction. For laparoscopic gastrectomy, end-to-side oesophagojejunostomy was performed using a $45 \mathrm{~mm}$ endoscopic linear stapler, followed by side-to-side jejunojejunal anastomosis using a $45 \mathrm{~mm}$ endoscopic linear stapler approximately $40 \mathrm{~cm}$ below the oesophagojejunal junction. For laparoscopic subtotal gastrectomy, gastrojejunostomy and end-to-side jejunojejunal anastomosis were performed using $45 \mathrm{~mm}$ and/or $60 \mathrm{~mm}$ linear staplers. The specimen was put into a special bag and extracted through the extended trocar incision.

The variables of short-term outcome included operative time, harvested lymph nodes, intraoperative and postoperative complications, duration of hospital stay, and 30-day mortality rate. The long-term outcome included disease-free survival time.

The patients with clinically (by CT scan) identified stage $\geq$ T2N1 underwent neoadjuvant chemotherapy. Patients received a combined intravenous cisplatin $80 \mathrm{mg} / \mathrm{m}^{2}$ once a day and capecitabin $1000 \mathrm{mg} / \mathrm{m}^{2}$ orally twice a day. The same scheme of adjuvant chemotherapy was given to patients with pathologically identified stage $\geq I$.

Statistical analysis was performed using SPSS 14.0 for Windows (SPSS Inc., Chicago, USA). The data is presented as mean \pm standard deviation or median and range. The cumulative survival was determined by the Kaplan-Meier method, and univariate comparisons between the groups were performed using the log-rank test. For comparison between groups, the Mann-Whitney test or Student's t-test were employed where appropriate. $P<0.05$ was considered statistically significant.

One hundred and seventy-five patients with proven gastric adenocarcinoma underwent laparoscopic or open radical gastric cancer surgery with D2 lymphadenectomy during the analysed period. Thirty-nine patients were unavailable for long-term follow-up. The most common reason for failed follow-up was change of residence (moving abroad). Data from 136 patients who were followed up postoperatively were analysed. The distribution of patients between groups and subgroups is shown in Figure 1.

There were no significant differences between the groups in term of gender, ASA score, and rates of administered neoadjuvant and adjuvant treatment. On the other hand, laparoscopic surgery was performed to significantly younger patients. Based on pathological data, the majority of Stage II-III tumours were removed. There was no significant difference $(p=0.426)$ in the distribution of disease stages between the 2 groups (Table I). It is interesting to note that significantly more diffuse type (according to Lauren) carcinomas were operated in a laparoscopic manner (Table I).

The average operating time was significantly longer in the LRS group; however, postoperative hospital stay was significantly shorter in the LRS group (Table II). There was no statistically significant difference in the number of harvested lymph nodes between both groups (Table II). Regarding major postoperative complications, the rates of oesophagojejunal anastomotic leakage ( $p=$ 0.497), intra-abdominal abscess ( $p=0.880$ ), pancreatic fistula ( $p=0.457)$, or postoperative bleeding $(p=0.258)$ did not present significant differences between the 2 groups. There were no conversions to open surgery in the LRS group. We had 7 (7.3\%) relaparotomies in the 


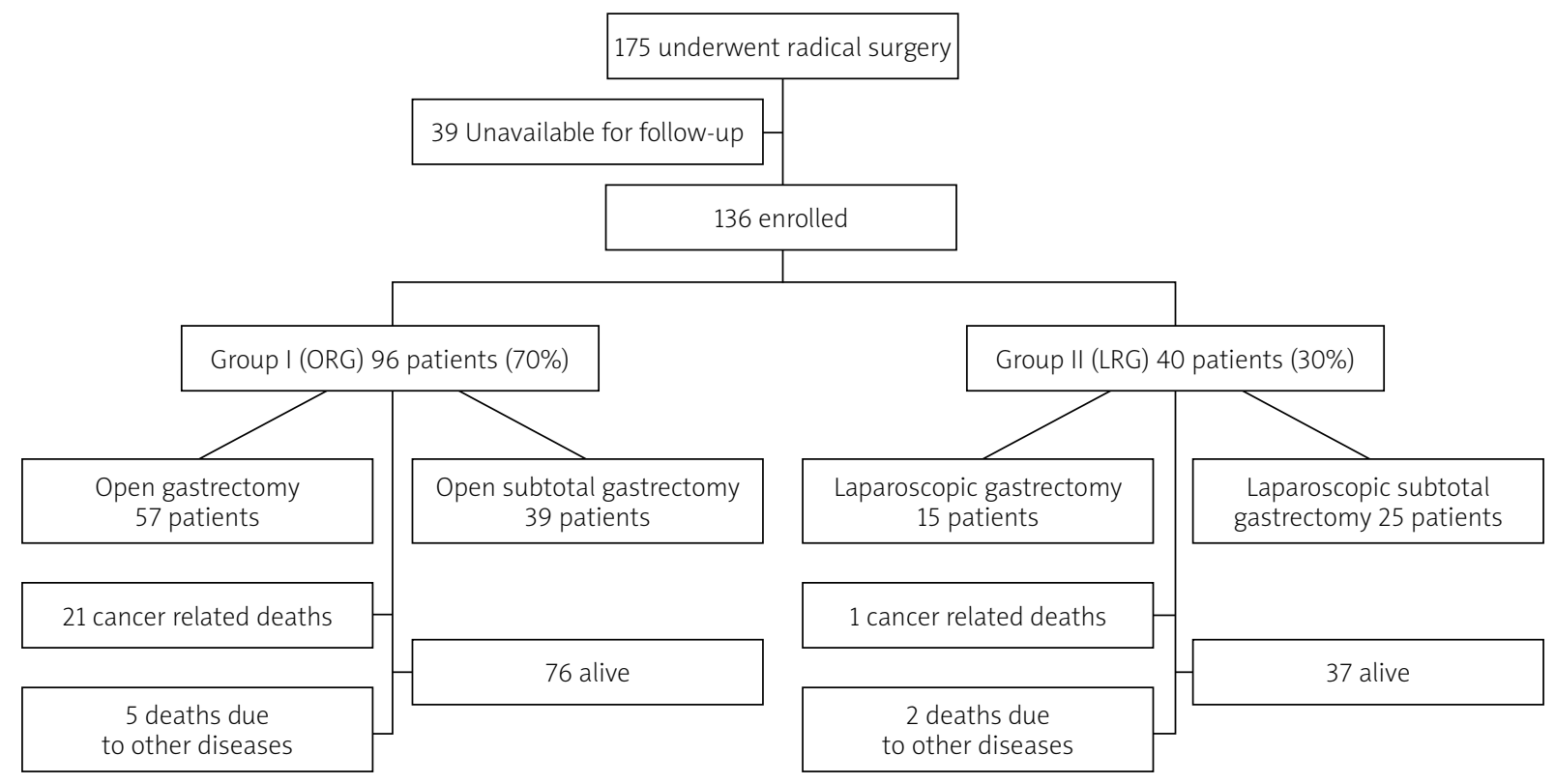

Figure 1. Patient's distribution between groups and subgroups

Table I. Patients' general characteristics

\begin{tabular}{|c|c|c|c|}
\hline Parameter & $\begin{array}{l}\text { Group I (ORS) } \\
\quad(N=96)\end{array}$ & $\begin{array}{l}\text { Group II (LRS) } \\
\quad(N=40)\end{array}$ & $P$-value \\
\hline \multicolumn{4}{|l|}{ Gender: } \\
\hline Male & $59(61.5 \%)$ & $23(57.5 \%)$ & 0.667 \\
\hline Female & $37(38.5 \%)$ & $17(42.5 \%)$ & 0.597 \\
\hline Age [years] & $67.4 \pm 11.1$ & $63.1 \pm 11.6$ & 0.042 \\
\hline \multicolumn{3}{|l|}{ ASA score: } & \multirow[t]{5}{*}{0.704} \\
\hline 1 & $1(1.0 \%)$ & $0(0.0 \%)$ & \\
\hline$\|$ & $21(21.9 \%)$ & $8(20.0 \%)$ & \\
\hline III & $68(70.8 \%)$ & $31(77.5 \%)$ & \\
\hline IV & $6(6.3 \%)$ & $1(2.5 \%)$ & \\
\hline Neoadjuvant treatment & $23(24.0 \%)$ & $10(25.0 \%)$ & 0.897 \\
\hline Adjuvant treatment & $52(54.2 \%)$ & $22(55 \%)$ & 0.929 \\
\hline \multicolumn{4}{|l|}{ Procedure: } \\
\hline Total gastrectomy & $57(59 \%)$ & $15(38 \%)$ & 0.066 \\
\hline Subtotal gastrectomy & $39(41 \%)$ & $25(62 \%)$ & 0.144 \\
\hline \multicolumn{4}{|c|}{ Histologic type (Lauren classification): } \\
\hline Intestinal & $48(50.0 \%)$ & $8(20.0 \%)$ & 0.005 \\
\hline Diffuse & $29(30.2 \%)$ & $19(47.5 \%)$ & 0.005 \\
\hline Mixed & $19(19.8 \%)$ & $13(32.5 \%)$ & 0.001 \\
\hline \multicolumn{3}{|l|}{ Pathologic stage: } & \multirow[t]{7}{*}{0.426} \\
\hline IB & $28(29.2 \%)$ & $15(37.5 \%)$ & \\
\hline$\| A$ & $12(12.5 \%)$ & $6(15.0 \%)$ & \\
\hline$\| B$ & $15(15.6 \%)$ & $8(20.0 \%)$ & \\
\hline IIIA & $13(13.5 \%)$ & $3(7.5 \%)$ & \\
\hline IIIB & $16(16.7 \%)$ & $5(12.5 \%)$ & \\
\hline IIIC & $12(12.5 \%)$ & $3(7.5 \%)$ & \\
\hline
\end{tabular}


Table II. Comparison of postoperative outcomes between ORS and LRS

\begin{tabular}{|c|c|c|c|}
\hline Parameter & $\begin{array}{l}\text { Group I (ORS) } \\
\qquad(N=96)\end{array}$ & $\begin{array}{l}\text { Group II (LRS) } \\
\quad(N=40)\end{array}$ & $P$-value \\
\hline Surgery time [min] & $234.4 \pm 50.9$ & $279.0 \pm 78.2$ & 0.002 \\
\hline Number of lymph nodes harvested & $25.9 \pm 11.6$ & $25.1 \pm 9.6$ & 0.756 \\
\hline Postoperative hospital stay [days] & $12.8 \pm 9.5$ & $9.7 \pm 5.8$ & 0.000 \\
\hline Postoperative morbidity & $34(35.4 \%)$ & $8(20 \%)$ & 0.196 \\
\hline \multicolumn{4}{|l|}{ Major postoperative complications: } \\
\hline Oesophagojejuno anastomotic leakage & $7(7.3 \%)$ & $2(5.0 \%)$ & 0.497 \\
\hline Intraabdominal abscess & $2(2.1 \%)$ & $1(2.5 \%)$ & 0.880 \\
\hline Postoperative bleeding & $3(3.1 \%)$ & 0 & 0.258 \\
\hline Pancreatic fistula & $2(2.1 \%)$ & 0 & 0.457 \\
\hline \multicolumn{4}{|l|}{ Reoperation: } \\
\hline (Relaparotomy/re-laparoscopy) & $7(7.3 \%)$ & $3(7.5 \%)$ & 0.971 \\
\hline \multicolumn{4}{|l|}{ Minor postoperative complications: } \\
\hline Surgical site infection (deep) & $2(2.1 \%)$ & 0 & 0.844 \\
\hline Pneumonia & $10(10.4 \%)$ & $1(2.5 \%)$ & 0.098 \\
\hline Urinal infection & $5(5.2 \%)$ & $3(7.5 \%)$ & 0.605 \\
\hline Other & $3(3.1 \%)$ & $1(2.5 \%)$ & 0.976 \\
\hline 30-day mortality & $4(4.2 \%)$ & $1(2.5 \%)$ & 0.846 \\
\hline
\end{tabular}

ORS group. Four of these were performed for postoperative peritonitis due to oesophagojejunal anastomotic leakage. Three relaparotomies were due to postoperative bleeding from the splenic artery pool on the first postoperative day. Three (7.5\%) re-laparoscopies were performed in the LRS group; 2 of these were due to oesophagojejunal anastomotic leakage, and 1 due to severe abdominal pain on the fifth postoperative day. An incarcerated Petersen's hernia was found and repaired laparoscopically.

There was no statistically significant difference in minor postoperative complications between the LRS and ORS groups (Table II). All complications in these groups were resolved without additional surgery.

There was no significant difference in 30-day mortality rate between the 2 groups (Table II).

The median follow-up period was 57 months (range: 25-86 months) in the ORG group and 45.5 months (range: 24-64 months) in the LRG group. In terms of overall survival, a total of 26 patients died after open surgery (27.1\%) and 3 after laparoscopic surgery (7.5\%), $p=0.018$, during the follow-up period (see the chart). The majority of cancer Stage III (33.3\%) and IV (38.1\%) patients died in the ORG group. In the LRG group, 1 patient died at cancer Stage II. Figure 2 shows disease-specific survival curves for all stages between the LRS and ORS groups with a significant difference in favour of the LRS group by long-rank test $(p=0.031)$.
Figure 3 shows disease-specific survival curves on I + II stages between the LRS and ORS groups with no significant difference $(p=0.550)$. Figure 4 shows disease-specific survival curves on III + IV stages between the LRS and ORS groups: the result almost reached a statistically significant difference $(p=0.051)$.

With the progression of minimally invasive surgery, laparoscopic gastric cancer surgery is receiving increasing attention from surgeons worldwide. Laparoscopic total or distal gastrectomy has been widely performed for patients with early gastric cancer in many countries. Brenkman et al. [11] in their international cross-sectional survey on gastric cancer surgery stated that a minimally invasive procedure was preferred for distal gastrectomy for early cancer (65\%). Moreover, surgeons in Asia also preferred a minimally invasive procedure for total gastrectomy for early cancer (63\%).

In the Netherlands, the uptake of minimally invasive gastric cancer surgery has increased from 4\% in 2010 to $43 \%$ in 2014 [12].

Our hospital is considered a high-volume centre for gastric cancer surgery, performing 70-80 radical surgeries (total or subtotal gastrectomies) each year. Routine curative procedure in the treatment of gastric cancer includes open or laparoscopic total or subtotal gastrectomy with D2 lymphadenectomy. Minimally invasive gastric cancer surgery was introduced in our clinic in 2005; however, between 2005 and 2015 only 1-2\% of 


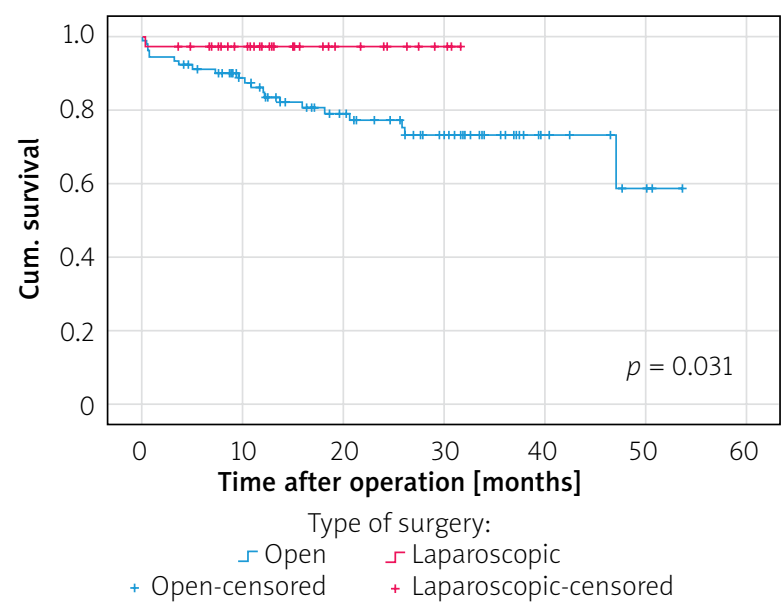

Figure 2. Overall disease specific survival time between ORG and LRG

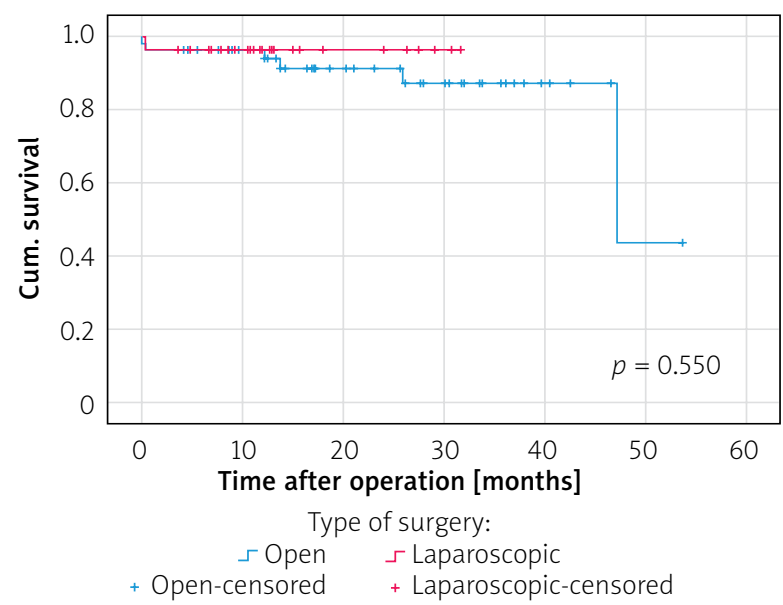

Figure 3. Disease specific survival time between ORG and LRG (Stage I + II)

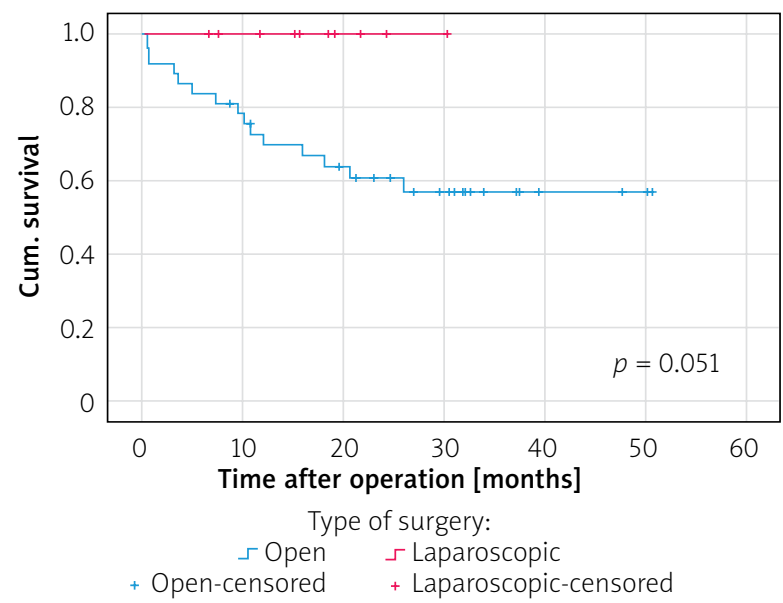

Figure 4. Disease specific survival time between ORG and LRG (Stage III + IV) gastric cancer surgeries were performed laparoscopically. The year 2015 was a turning point in laparoscopic gastric cancer surgery: improved funding led to a $55 \%$ increase in minimally invasive gastric cancer surgery cases by the year 2019.

Most laparoscopic gastric cancer surgery data are from East Asian countries (China, Japan, South Korea); however, direct projection of these results to Western populations is not always possible. Some differences between Eastern and Western populations possibly exist, such as the age and stage of the disease, tumour biology, and socio-epidemiological and ethnic characteristics [13].

Brenkman et al. [11] stated that the majority of surgeons (84.4\%) worldwide report the application of neoadjuvant chemotherapy or chemoradiation prior to gastrectomy. This study also reported that the percentage of surgeons in Europe (76.8\%) using neoadjuvant therapy (2013-2014) is higher compared to the period of $2011-2012$, when more than $60 \%$ of patients did not receive neoadjuvant therapy [14]. Our study demonstrated that during the period 2013-2017, only $24 \%$ of patients received neoadjuvant chemotherapy, without a significant difference between the groups. Compared with the above-mentioned data, this figure is very low. This can be explained by the fact that as a routine procedure, neoadjuvant chemotherapy was not initiated in our hospital until the beginning of 2015.

The majority of studies that compare open versus laparoscopic gastric cancer surgery examine total gastrectomy or subtotal gastrectomy groups separately. In our study we decided to analyse these groups (total gastrectomy and subtotal gastrectomy) together, due to the small number of patients in each group.

Our study results demonstrated no significant difference in the rate of major and minor postoperative complications, the number of harvested lymph nodes, or the 30-day mortality rate between the ORS and LRS groups. In their recent meta-analysis of open and laparoscopic gastrectomies, Zeng et al. [7] performed a subgroup analysis that was stratified based on different cancer stages (early gastric cancer and advanced gastric cancer) as well as different types of gastrectomy (distal gastrectomy). This subgroup analysis also did not find significant differences in lymph nodes harvested during surgery, severe complications, recurrence, or mortality between these 2 groups. In their population-based cohort study, Brenkman et al. [15] also concluded that minimally invasive gastrectomy is comparable to open gastrectomy in the context of postoperative morbidity, mortality, and harvested lymph nodes. On the other hand, Ludwig et al. [16] in a matched pair analysis study for advanced gastric cancer pointed out that total num- 
bers of retrieved lymph nodes were significantly higher in laparoscopic gastrectomy $(33.1,17-72)$ than in open gastrectomy $(28.2,14-57)$, and that the overall morbidity in the open gastrectomy group (44.4\%) was twice as high as in the laparoscopic gastrectomy group $(22.2 \%$, $p<0.05)$ due to a higher rate of minor complications.

Although there was no significant difference in oesophagojejunal anastomotic leakage rates between the LRS and ORS groups ( $12.3 \%$ vs. $13.3 \%)$, the overall oesophagojejunal anastomotic leakage rate was quite high in both groups. Reviewing results from Asia and West European countries [2, 7, 14-17], the incidence of oesophagojejunal anastomotic leakage varies from $4 \%$ to $8 \%$ in both laparoscopic and open gastrectomy groups. The high oesophagojejunal anastomotic leakage rates in our study can be explained by the relatively small number of consecutive patients (open gastrectomy - 57 pts, laparoscopic gastrectomy - 15 pts). In our previously published study [18], which analysed the results of gastric cancer treatment over 2 decades, the incidence of oesophagojejuno anastomotic leakage after open gastrectomy was $\sim 3 \%$.

The 30 -day mortality rate $(2.5-4.2 \%)$ recorded in our study is acceptable and comparable to the majority of published data [2, 7, 14-17].

As to the long-term outcome, the median follow-up period in our study reached 57 months in the ORG group and 45.5 months in the LRG group, and the overall cumulative survival rate had a significant difference in favour of the laparoscopic radical surgery group. These results should be assessed with great caution, because of the small number of patients in both groups, especially in the LRG group.

There are some serious limitations in this study: 1. This is a retrospective study as opposed to a randomised clinical trial, where the operation technique is chosen independently by the operating surgeon; 2. The number of patients in each group is relatively small, which limited our possibilities to independently analyse total and subtotal gastrectomy subgroups.

In conclusion, The results of our study demonstrated that LRG is comparable to ORG regarding postoperative morbidity, mortality, and long-term outcomes. Nevertheless, it results in a shorter hospital stay and may determine earlier initiation of adjuvant chemotherapy.

However, more high quality and sufficiently large sample randomized controlled trials are required to adequately compare short-term and long-term outcomes after laparoscopic and open gastrectomies, particularly in Europe.

\section{Acknowledgments}

We thank Mrs A. Smith for English language proofreading and editing services.

\section{Conflict of interest}

The authors declare no conflict of interest.

\section{References}

1. Bray F, Ferlay J, Soerjomataram I, et al. Global cancer statistics 2018: GLOBOCAN estimates of incidence and mortality worldwide for 36 cancers in 185 countries. CA Cancer J Clin 2018; 68: 394-424.

2. Russo A, Li P, Strong VE. Differences in the multimodal treatment of gastric cancer: East versus west. J Surg Oncol 2017; 115: 603-14.

3. Parkin DM, Bray F, Ferlay J, et al. Global cancer statistics, 2002. CA Cancer J Clin 2005; 55: 74-108.

4. Kurtinaitis J, Smailyte G. Survival of stomach cancer patients in Lithuania. Evalution of cases diagnosed in 1998-2000. Public Health 2005; 4: 67-71.

5. Smyth EC, Verheij M, Allum W, et al. Gastric cancer: ESMO Clinical Practice Guidelines for diagnosis, treatment and follow-up. Ann Oncol 2016; 27 (suppl 5): v38-49.

6. Japanese Gastric Cancer Association. Japanese gastric cancer treatment guidelines 2014 (ver. 4). Gastric Cancer 2017; 20: $1-19$.

7. Zeng F, Chen L, Liao M, et al. Laparoscopic versus open gastrectomy for gastric cancer. World J Surg Oncol 2020; 18: 20.

8. Wang Z, Xing J, Cai J, et al. Short-term surgical outcomes of laparoscopy-assisted versus open D2 distal gastrectomy for locally advanced gastric cancer in North China: a multicenter randomized controlled trial. Surg Endosc 2019; 33: 33-45.

9. Yu J, Huang C, Sun Y, et al. Effect of laparoscopic vs open distal gastrectomy on 3-year disease-free survival in patients with locally advanced gastric cancer. JAMA 2019; 321: 1983-92.

10. Japanese Gastric Cancer A. Japanese classification of gastric carcinoma: 3rd English edition. Gastric Cancer 2011; 14: 101-12.

11. Brenkman HJF, Haverkamp L, Ruurda JP, van Hillegersberg R. Worldwide practice in gastric cancer surgery. World J Gastroenterol 2016; 22: 4041-8.

12. Dutch Upper GI Cancer Audit. DUCA Jaarrapportage 2014. 2015. Available at: http://www.clinicalaudit.nl/jaarrapportage/2014/duca.html.

13. Strong VE, Song KY, Park CH, et al. Comparison of gastric cancer survival following $\mathrm{RO}$ resection in the United States and Korea using an internationally validated Nomogram. Ann Surg 2010; 251: 640-6.

14. Messager M, de Steur WO, van Sandick JW, et al. Variations among 5 European countries for curative treatment of resectable oesophageal and gastric cancer: a survey from the EURECCA Upper GI Group (EUropean REgistration of Cancer CAre). Eur J Surg Oncol 2016; 42: 116-22.

15. Brenkman HJF, Gisbertz SS, Slaman AE, et al. Postoperative outcomes of minimally invasive gastrectomy versus open gastrectomy during the early introduction of minimally invasive gastrectomy in the Netherlands: a population-based cohort study. Ann Surg 2017; 266: 831-8.

16. Ludwig K, Schneider-Koriath S, Scharlau U, et al. Totally laparoscopic versus open gastrectomy for gastric cancer: a matched pair analysis. Zentralbl Chir 2018; 143: 145-54. 
17. Shida A, Mitsumori N, Fujioka S, et al. Comparison of shortterm and long-term clinical outcomes between laparoscopic and open total gastrectomy for patients with gastric cancer. Surg Laparosc Endosc Percutan Tech 2016; 26: 319-23.

18. Mickevicius A, Ignatavicius P, Markelis R et al. Trends and results in treatment of gastric cancer over last two decades at single East European centre: a cohort study. BMC Surgery 2014; 14: 98.

Received: 29.10.2020

Accepted: 2.12 .2020 